\title{
Multi-Region Data Simulation for Detection Limit Investigations in IVAS ${ }^{\text {TM }}$
}

\author{
R. M. Ulfig ${ }^{1}$, B. P. Geiser ${ }^{1}$, T. J. Prosa ${ }^{1}$, D. J. Larson ${ }^{1}$, J. Leroux ${ }^{2}$, and B. Dupont ${ }^{2}$ \\ 1. CAMECA Instruments, Inc. 5500 Nobel Drive, Madison, WI 53711 USA \\ 2. CAMECA SAS 29 Quai des Gresillons, 92622 Gennevilliers, Cedex France
}

Atom Probe Tomography, (APT) is the highest spatial resolution analytical technique and due to recent advances in commercially available instruments and sample preparation techniques, APT is being used on a variety of increasingly challenging three-dimensional (3D) applications [1]. Predicting the detection and quantification limits of low concentration constituents can be challenging from a practical point of view, but is critical when one need to answer what applications of APT are feasible. The new Region of Interest, (ROI) simulation tools included in Integrated Visualization and Analysis Software (IVAS) version 3.6.6 allow a relatively straight forward means to 1) estimate detection limits and 2) use actual analytical tools such as concentration profiles, proximity histograms and two-dimensional concentration maps, etc., on simulated, but spectrally realistic data. Figure 1 demonstrates the ability to create realistic mass spectral and 3D position data as compared to an actual doped silicon analysis.

The initial implementation of the ROI simulation tool allows multiple layers to be created with each layer having a specified signal to noise ratio (SNR) and an arbitrary number of constituents (each constituent can have an arbitrary peak shape mass resolving power (MRP) and specified charge state ratio). The resultant simulated 3D dataset can be analysed and detection limits can be calculated. The case of dopants in microelectronic structures provides an example of a realistic simulation providing direction in predicting detectability and quantification. In bulk single crystal silicon one can often use analytical conditions that maximize data quality (MRP and SNR) to extend the detectability of dopants to near part per million levels. For complex nanoscale structures that are analysed at lower detection rates to minimize sample fracture, the resultant SNR, MRP, and peak shapes can be degraded, impacting detection sensitivity. A priori knowledge of the required spectral quality to achieve detection or quantification is key to understanding what experimental conditions need to be optimized to efficiently achieve an experimental goal or to know when that goal is practically unachievable.

A series of simulations of dopants in silicon were created using the ROI Simulation Tool in IVAS, each with varying levels of mass resolving power and background noise level to observe the effects on detection limits. Upper and lower peak range limits were chosen at twice the local background level. Fig. 2 shows the variation of detection limit of boron-11 (95\% confidence that a peak exists [2] ) as a function of MRP and noise level over experimentally realistic values for such data collected on a LEAP $4000 \mathrm{X}^{\mathrm{TM}}$ system (MRP of 500-1800 and Noise metric 5-60 ppm/nsec). It is clear from Fig. 2 that over experimentally realistic values, minimization of the background noise level is the more important parameter for the minimization of the detection limit of a small concentration isolated ion.

The ROI Simulation Tool can create multi-layered structures with arbitrary constituents, concentrations, peak shapes, and noise levels. The simulated volumes can be analysed much like any other APT file in the new POS Analysis feature in IVAS using the same analysis tools and the same 3D display. Figure 3 shows such an example of a 3D dataset displayed, and analysed with a proximity histogram and volume rendering of the boron distribution. Analysing simulated corollaries of actual analysed structures allow one to understand how ranging, voxel size, and delocalization affect data interpretation. Although in the simulated data, the interfaces are discrete and constituent distribution random, concentration profiles and $3 \mathrm{D}$ concentration rendering show variation and non-zero interface widths that could be misinterpreted. 
References:

[1] T. F. Kelly and D. J. Larson, Annual Reviews of Materials Research 42 (2012), p. 1.

[2] L.A. Currie, Limits for Qualitative Detection and Quantitative Determination, Anal. Chem. 40 (1968) p. 586.

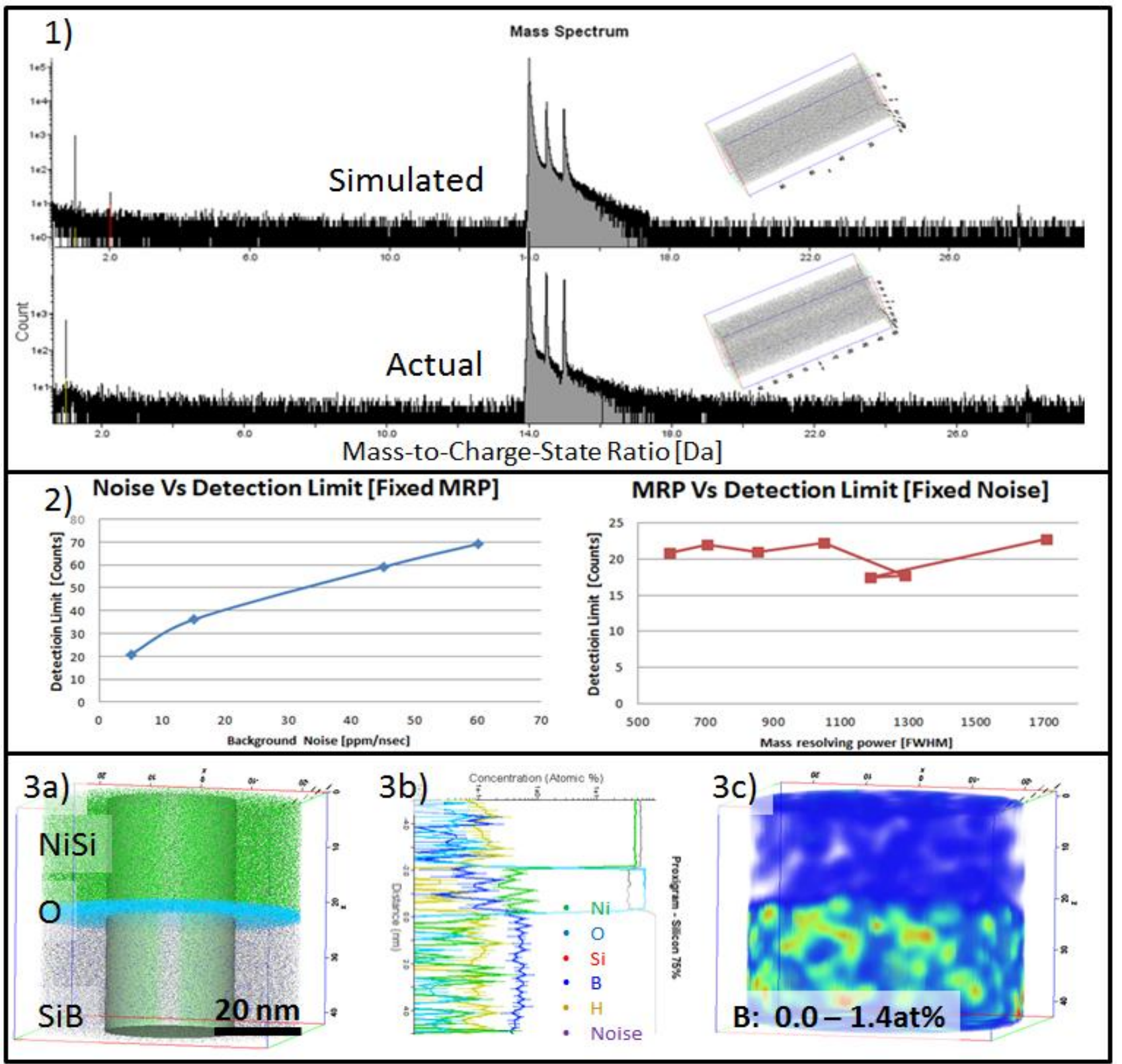

Figure 1. Overlay of an actual and simulated mass spectrum and 3D position file of an antimony doped silicon analysis on a LEAP 4000X system.

Figure 2. Detection limit for boron-10 as a function of background noise and Mass Resolving Power demonstrating that in this analysis, MRP has little effect upon sensitivity low concentration species.

Figure 3. Example analysis of a multilayer simulated dataset with: a) 3D position file, b) 1D proximity histogram, and c) a volume rendering of boron concentration. Note how a random distribution of boron in a volumetric analysis shows apparent clustering. 